NASA-TM-112168

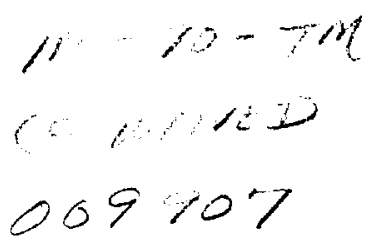

\title{
LABORATORY STUDIES OF DIB CARRIERS
}

\author{
L.J. Allamandola \\ NASA Ames Research Center, MS 245-6 \\ Moffett Field, CA 94035-1000
}

\begin{abstract}
Spectroscopic studies of the following potential diffuse interstellar band (DIB) carriers are reviewed: unspecified organics, carbon chains, polycyclic aromatic hydrocarbons (PAHs), fullerenes and derivatives, as well as porphyrius and related material. An assessment of each is given, along with suggestions for further experimental studies needed to fully test cach candidate. Of the experimental techniques in common use, matrix isolation spectroscopy with neon matrices is the most appropriate for the DIBs. The low vapor pressure and high reactivity of these naterials preclude gas phase studies on many of these species. At this point, given the type and quality of published data available, carbon chains and PAHs are the nost promising candidates for a number of the DIBs.
\end{abstract}

\section{INTRODUCTION}

Identifying the cauriers of the diffuse interstellar bands (DIBs) has becone the classic: spectroscopic problem of the 20th Century. Since their discovery in the carly 1900's the DIBs have successfully challenged spectroscopists, astronomers and pliysicists. During this time so many suggestions have been made, experiments carried out, and theories proposed that a thorough review would more than fill this volume. The same holds true for a summary of experiments on species which might be relevant to the DIB problem. Here, the focus will rather be placed on a review of the experiments which were specifically carried out to address the DIB question, with the further restriction that the types of materials discussed represent what are currently thought to be promising candidates. Even with this, the scope is large. Many researchers active in this area have been asked for relevant references and cross references in order to be as thorough as possible. Nontheless, some important contributions have almost certainly been overlooked.

The experimental challenge was succinctly stated by Johnson (1970) twenty five vears ago, "... one not only has to match 25 diffuse interstellar lines as far as wavelengths are concerned, but also as far as intensity. In addition, the... interstellar line widths vary from $40 \AA$ to $1 \AA$...and are invariant to $0.1 \AA$..." Since that time the number of DIBs has grown to well over 100 , their relative intensitics have been shown to vary from one line-of-sight to another, and some DIBs seem to go together in loosely connected families (Herbig 1975; Jenniskens and Désert 1994: Lrefowski and Walker 1987). Wavelength invariance has been taken to indicate that the carriers cannot reside in or on dust particles. Thes is because particle size, shape aurd composition influence peak position and profile, and it is difficult to imagine that

175

A. G. G. M. Tielens and T. P. Snow (eds.), The Diffuse Interstellar Bands, 175-198. (c) 1995 Kluwer Academic Publishers. Printed in the Netherlands. 
the grains along all lines-of-sight are exactly the same. Thus, while this is used to further limit the work reviewed here to molecular sized species in the gas phase, one must keep in mind that any sweeping conclusions based on global DIB observational properties are probably invalid. This is intrinsic to the DIB problem. Quite often generalizations are based on studies of only a handful of the strongest DIBs. There is good reason for focusing on these, as most DIBs are weak and inherently difficult to measure. Nonetheless, these strong DIBs are certainly not representative of the entire collection along all lines-of-sight.

The criteria which must be met for a particular material to be accepted as a DIB carrier are that its visible and near-infrared absorption features match the known DIBs in wavelength, bandwidth and relative intensities, while not possessing additional features that are absent in the interstellar spectra. There are no species which satisfy these criteria to date, there are only candidates. Experimentalists are charged with measuring the spectra of candidate materials which can be compared with the DIBs. Ultimately, one strives to obtain spectra which can be directly compared to the DIB spectrum. Ideally, this requires measuring the absorption spectra of various candidates in the gas phase at interstellar temperatures and pressures. This also includes determining the oscillator strength for each transition, as this is needed to derive the column density necessary to produce the observed equivalent width. Practically, since many of the promising species are difficult to work with, these goals are extremely challenging, if not impossible, given current experimental capabilities.

These issues are discussed in this review. First, experimental techniques are summarized, pointing out the inherent advantages and disadvantages of each approach. Next, specific examples are given of species which are currently considered to be promising as contributors to the diffuse interstellar bands. Finally, an assessment of these candidates as potential DIB carriers is given along with suggestions for the experiments needed to provide additional tests for species.

\section{EXPERIMENTAL TECHNIQUES}

On face value, measuring DIB candidate absorption spectra is simple enough. One needs a visible light source, a monochromator, and a detector. This equipment is readily available. The experimental challenge often lies in sample preparation and, during the time required to measure it's spectrum, maintenance. This is because the most promising candidate materials are either refractory or, due to the harsh interstellar radiation environment, short lived under most terrestrial conditions. Thus, the experimentalist must be able to suspend individual refractory molecules in a manner which minimizes or eliminates any perturbation from the surrounding medium, or prepare and store reactive species unperturbed for periods long enough to permit the measurement of their spectra.

The advantages and disadvantages of the sample preparation techniques which are in common spectroscopic use are summarized in Table 1 . The first three, while straightforward, are not appropriate for measuring the spectra of isolated, individual species. In all three - ground powders, liquid solutions, or pellets (transparent solids containing suspensions of the material of interest) - interactions with the host material can severely perturb the transitions in the guest. These perturbations cause shifts in band positions, intensities and profiles. Thus, while these methods are useful as an initial screen to identify promising candidates, they cannot be used in one-on-one comparisons with the DIBs. The last two methods - matrix isolation and gas phase spectroscopy - provide data which is more appropriate for the DIB issue. 
Table 1

Advantages and disadvantages of sample preparation techniques

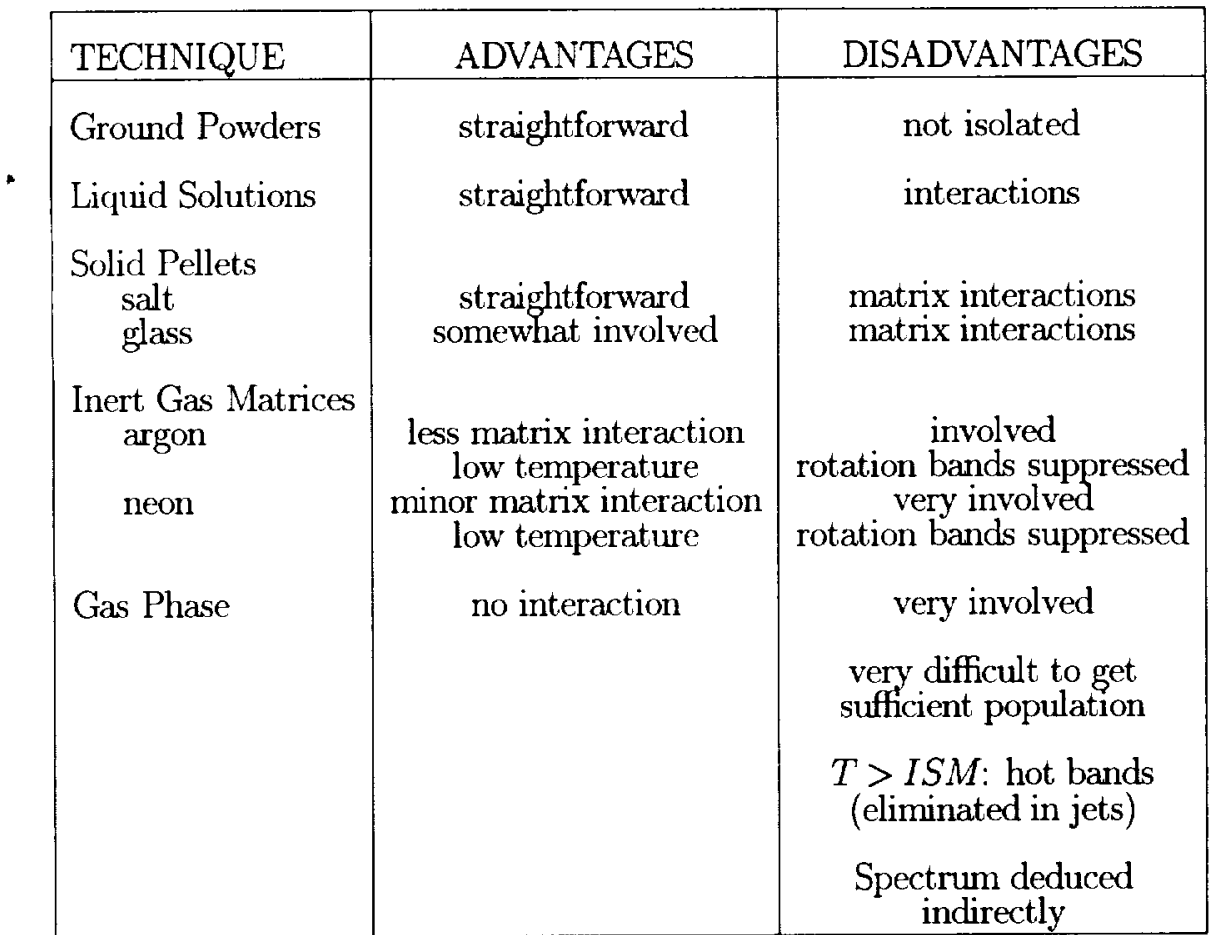

Matrix Isolation Spectroscopy and the DIBs- Matrix isolation spectroscopy, developed by Professor Pimentel and his colleagues in the 1950's, is the technique in which the species of interest is suspended in a frozen inert gas matrix. This is ideally suited to the challenges provided by both refractory and reactive DIB candidates. Briefly, the technique consists of simultaneously codepositing the species of interest with an overabundance of inert gas such as neon, argon, or krypton onto a cold window which is suspended in a vacuum chamber. The vacuum should lie in the high- to ultra-high range $\left(10^{-7}-10^{-8} \mathrm{mbar}\right)$. Such high vacuum conditions, as well as very pure starting materials ( $>99.5 \%$ for the guest, $99.999 \%$ for the host), are essential to ensure that all the spectral features obtained can be confidently attributed to the target species. This is especially true in the cases where the species is a reactive intermediate produced by some form of energetic alteration of the starting materials. Here processes such as irradiation or electron bombardment can produce many new and exotic species. It is often a daunting task to identify the precise chemical identity of the species in such matrices under the best of conditions. Thus, while often glossed over, it is essential that great care be given to the mundane task of sample preparation. The deposition rate must be slow and the window temperature quite low to ensure that the gases freeze out quickly, without allowing diffusion to occur. The rapid formation of a rigid matrix is required since any diffusion will result in the production and trapping of aggregates, as well as isolated species; or promote rcactions between reactive species such as ions and radicals, resulting in the loss of 
the target species and the production of unwanted reaction products. Both processes produce spurious spectral features. The maximum window temperature acceptable is determined by the inert gas, with temperatures below about $20 \mathrm{~K}$ sufficient for argon and below about $5 \mathrm{~K}$ required for neon. Since $10 \mathrm{~K}$ is the minimum temperature mechanical coolers can currently achieve with reasonable cooling powers, argon matrices are commonly employed. However, due to the fact that the band shifts from gas phase values are greater in argon matrices than neon matrices, neon is required for the DIB problem, a point discussed further below. Thus, liquid helium coolant (and all the care it entails) is required to produce the matrices which are most appropriate for the DIB issue. Once the matrix is produced, absorption spectra are measured using customary techniques. These data are then compared to the DIBs.

The key question here is, "How relevant is neon matrix data for the DIB issue?" Neon matrices perturb transitions, resulting in band shifting and broadening. Relative band strengths may also be slightly affected. The material is frozen in a solid, thus eliminating the rovibrational structure inherent in a gas phase spectrum, and trapping in different sites can produce spurious spectral features. Thus, one wonders if neon matrix spectra are at all relevant. They are very relevant. These matrices are used because it is exceedingly difficult (if not impossible) to prepare and study the gas phase spectra of many serious DIB candidate materials (see below) and, of all known sample preparation techniques, the spectra of materials suspended in solid neon have been shown to provide the best wavelength matches to the bands in the gas phase spectra of the same species. Thus, at the very least, neon matrices provide a means by which many candidate materials, some exceedingly difficult to work with, can be screened.

The situation is actually much better, and serious DIB candidates can be identified using neon matrix isolation spectroscopy (See, for example, the articles by Maier, Salama, and d'Hendecourt elsewhere in this volume). In neon, the shifts are very small, broadening is not severe, the $0-0$ band position is often readily discernable, and site splitting is generally not a problem. While gas phase profiles are not duplicated in neon, one can calculate reasonably accuraté rotational band envelopes. The tables of shifts between the gas phase and neon matrix frequencies for a particular ion transition listed by Bondybey and Miller (1983) illustrate this point. The ions listed include diacetylene, hexatriene, the aromatic molecule berzene $\left(\mathrm{C}_{6} \mathrm{H}_{6}\right)$ and many halogen, hydroxy, and alkyl substituted benzenes. Less than $0.5 \%$ frequency shifts $\left(\left(\left(\nu_{\mathrm{g}}-\nu_{\mathrm{n}}\right) / \nu_{\mathrm{g}}\right) \times 100\right)$ are reported for the majority of bands listed (15 out of 26$)$. Eight of the remaining nine are shifted by less than $1.0 \%$, with the largest occurring in species containing chlorine and bromine. For the transitions having less than a $0.5 \%$ shift, the average difference between the neon matrix and gas phase values is $66 \mathrm{~cm}^{-1}$, with the neon data always shifted to lower frequencies. For those less than $1 \%$, the average is $94 \mathrm{~cm}^{-1}$. Therefore, the criterion for a material to be seriously considered as a plausible DIB candidate is straightforward. The band frequencies of the species, when isolated in a neon matrix, must fall within $1 \%$ that of a DIB frequency. A smaller shift is required for neutral species for the same confidence level. Bondybey and Miller (1983) also show that the upper state lifetimes for a particular ionic species isolated in neon are very close to the gas phase values, allowing one to deduce gas phase band widths from neon matrix data as woll. The reason that neon is particularly useful, especially for ionized species, arises from its $24.6 \mathrm{eV}$ ionization potential as compared to the $15.76 \mathrm{eV}$ ionzation potential for argon. Much less charge transfer between guest and host can take place in neon, resulting in nuch weaker interactions (Bondybey and Miller, 1983). 
Gas Phase Spectroscopy and the DIBs- Gas phase absorption spectroscopy of many suitable candidate materials remains in the wishful thinking category. While it is often expressed that the final test awaits gas phase data on the species of interest taken under the appropriate conditions, the extreme difficulties associated with attaining this obvious goal are not at all appreciated by many in this field. There are several severe stumbling blocks which must be overcome. While it is exciting to realize that this type of data may become available on a few systems during the next few years, unless there is a significant experimental breakthrough, it is unlikely that many - systems will be so studied. Here, the challenges have several sources.

Although molecular beam techniques employing jets can generate cold samples in a collision-free environment, ideal conditions for mimicking the interstellar modium, these jets generally do not contain enough material to pernit an absorption spectrum measiuement over a narrow spectral region, let alone across the entire LV-Visible-near IR range. Most jet experiments take advantage of the extreme sensitivity of the laserinduced-fluorescence technique, which provides high resolution emission spectra over a very narrow spectral range. In order to maintain sufficient colum densities of the individual molecular constituents of the refractory material of interest to permit the measurement of an absorption spectrum over a broad spectral range requires sauple cell temperatures significantly higher than relevant for the ISM. Thus, low lying states are populated and the absorption spectrum must be corrected appropriately. As the molecular spectroscopic parameters required to accurately and precisely carry out such an analysis are poorly known for many of the species of interest, simplifying a high temperature spectrum will introduce uncertainties in band positions, profiles, and relative intensities. These difficulties apply to reactive species as well, which carry the additional burden of sample maintenance. Even for refractory species which do not need very high temperatures, sample generation often requires the sudden input of energy and the species are not prepared in their ground state, once again requiring spectral deconvolution. Thus, while it secms reasonable to presume that this type of data will be obtained for a few DIB candidates within several years, it is not likely that spectra of the large number of species that must be measured to adequately address the more than 100 DIBs will be available for some time to come. Sec Boissel (1995, elsewhere in this volume) for the description of a novel techmiguc which may ultimately yield moderate resolution spectra of volatile, reactive DIB candidates. (The author hopes he is overly pessimistic here, and that this critique stimulates a fow spectroscopists to prove him wrong.)

Consequently, neon matrix isolation spectroscopy, in spite of it's limitations, remains the most promising technique to use. It offers the ability to survey nany possibilities in a reasonably short period of time providing data that is directly applicable to the DIB problem.

Apart from the spectroscopic studies discussed here, additional experimental work is also called for. Properties such as ionization potentials and cross sections, photodissociation rates and cross sections, electron affinities, electron recombination rates aud so forth are required for the most promising candidates. With this information onc can ascertain which forms are most likely to be present in various regions of the (liffuse interstellar medium. Since the spectrum of a species changes with ionization state, these data give much needed supplemental insight into the DIB problem. For example, knowledge of these properties enables one to determine the fraction of a given species that will be in its cationic $(+)$, anionic $(-)$, and nentral fonns, and which species are likely to survive interstellar conditions. Leach (1995, elsewhere in this volime) has reviewed the available data on this subject. 


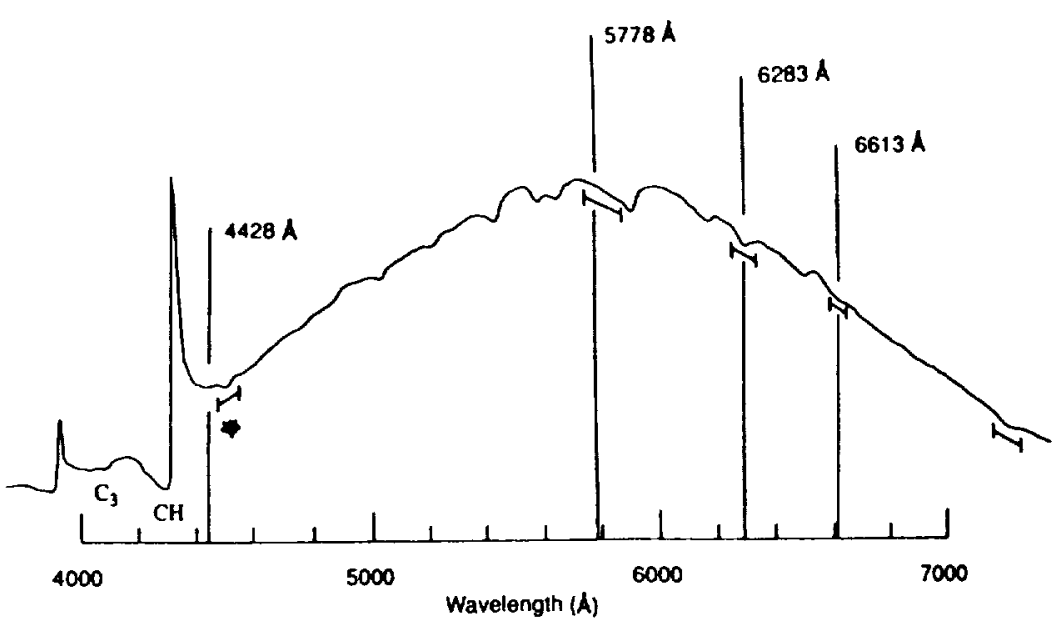

Fig. 1. Single beam absorption spectrum of an argon matrix containing the discharge products from an $\mathrm{Ar}: \mathrm{CH}_{4}(200: 1)$ mixture which has been irradiated with ultraviolet light. Vertical lines indicate the positions of 4 of the strongest DIBs. The broad laboratory absorption features proposed as possible DIB counterparts are also indicated (Wdowiak, Lee, and Beegle 1993). Table 2 lists the peak absorption wavelengths of the DIB candidates.

Table 2

Absorptions produced in an $\mathrm{Ar}: \mathrm{CH}_{4}(200: 1)$ discharge compared to the $\mathrm{DIBs}^{b}$

\begin{tabular}{|c|c|c|c|c|c|}
\hline & & \multicolumn{4}{|c|}{ Correlated Laboratory Feature } \\
\hline \multicolumn{2}{|c|}{ DIB } & \multirow[b]{2}{*}{ Feature } & \multirow[b]{2}{*}{$\lambda(\AA)$} & & \multirow{2}{*}{$\frac{\nu_{\mathrm{DIB}}-\nu_{\mathrm{L}_{\mathrm{AB}}}}{\nu_{\mathrm{DIB}}} \times 100 \%$} \\
\hline$\lambda(A)$ & $\mathrm{cm}^{-1}$ & & & $\mathrm{~cm}^{-1}$ & \\
\hline $\begin{array}{c}4430 \\
4754,4763 \\
5535 \\
5705 \\
5779 \\
6269 \\
6283 \\
6613 \\
6992 \\
7223\end{array}$ & $\begin{array}{c}22573 \\
21025,20989 \\
18062 \\
17523 \\
17304 \\
15952 \\
15916 \\
15122 \\
14302 \\
13845\end{array}$ & $\begin{array}{l}\text { a } \\
g \\
f \\
d \\
c \\
b \\
b \\
e \\
I R \\
\text { IR }\end{array}$ & $\begin{array}{l}4500 \\
4755 \\
5587 \\
5687 \\
5788 \\
6271 \\
6279 \\
6601 \\
6882 \\
7194\end{array}$ & $\begin{array}{l}22220 \\
21030 \\
17900 \\
17583 \\
17277 \\
15946 \\
15926 \\
15150 \\
14530 \\
13900\end{array}$ & $\begin{array}{c}+1.57 \\
-0.02,-0.20 \\
+0.90 \\
-0.34 \\
+0.16 \\
+0.03 \\
-0.06 \\
-0.19 \\
-1.59 \\
-0.40\end{array}$ \\
\hline
\end{tabular}

a Attributed to matrix shifts.

${ }^{b}$ Features labeled a, g... are discussed in the original paper (Wdowiak 1980). 


\section{SPECIFIC EXAMPLES OF DIB CANDIDATES}

The following materials are currently considered as potential DIB carriers.

-Unspecified Organics

- Carbon Chains

- Polycyclic Aromatic Hydrocarbons

- Fullerenes and Their Derivatives

- Porphyn/Porphyrins

All contain the cosmically abundant element carbon. Since these are molecules, not grains, the goal has been to obtain spectra of the isolated species. As will become clear in the following discussion, while some may be more attractive than others as potential contributors to the DIBs, none satisfy all the criteria summarized by Snow near the end of this book. Each will now be discussed in turn.

\subsection{UNSPECIFIED ORGANICS}

In 1980 Wdowiak produced candidates for the carriers of several DIBs in argon matrix experiments. Samples were prepared by passing an $\mathrm{Ar}: \mathrm{CH}_{4}(200: 1)$ mixture through an electric discharge and condensing the products onto a sapphire window $(\sim 12 \mathrm{~K})$. In addition to features arising from several well known species such as $\mathrm{C}_{2}^{-} \cdot \mathrm{CNN}$, and $\mathrm{CH}$, a few new bands were evident as shown in Figure 1. Several (see Fig. 1 and Table 2) fell within reasonable matrix shifts of DIBs. The bands were photostable under ultraviolet radiation suggesting that the carriers could survive the interstellar radiation field. While several fall near the strong DIBs at 5778,6283 , and $6613 \AA$, the strongest, a broad absorption band near $4500 \AA$, is particularly interesting. It becomes evident only after $\mathrm{CH}_{3} \mathrm{C}_{2}^{-}$and $\mathrm{C}_{3}$ bands are strong, suggesting it arises from a species containing several carbon atoms. This is supported by the observation that a similar band is produced in pure carbon vapor deposition experiments (next section). These experiments have been repeated and subjected to further analysis as described by Wdowiak et al. (1994).

\subsection{CARBON CHAINS}

Krätschmer (1986) investigated the possibility that carbon chains might be responsible for the DIBs by preparing argon matrices containing small carbon molecules and atoms. These matrices were produced by depositing a stream of argon onto a cold sapphire window $(10 \mathrm{~K})$ simultaneously with carbon vapor generated from a resistively heated carbon rod. The ratio of argon to trapped species (100 to 1000) was controlled by adjusting the relative deposition rates. This produced an argon matrix containing small carbon species such as $\mathrm{C}, \mathrm{C}_{2}$, and $\mathrm{C}_{3}$. As shown in the lower trace of Figure 2, these absorb below about $4200 \AA$. By warming the matrix slightly, these smaller species can diffuse and react with each other producing larger carbon molecules (Figure 2). These often absorb at longer wavelengths. For example, the strong $4100 \AA$ absorption assigned to $\mathrm{C}_{3}$ decreases while bands at $3110,3480,3940$, $4470 \AA$ and longer wavelengths grow in. That at $4470 \AA$ was tentatively assigned to $C_{7}$. Tentative assignments of some of the longer wavelength bands are shown in Figure 3 . The bands seem uncorrelated, indicating that each arises from a different molecule. 


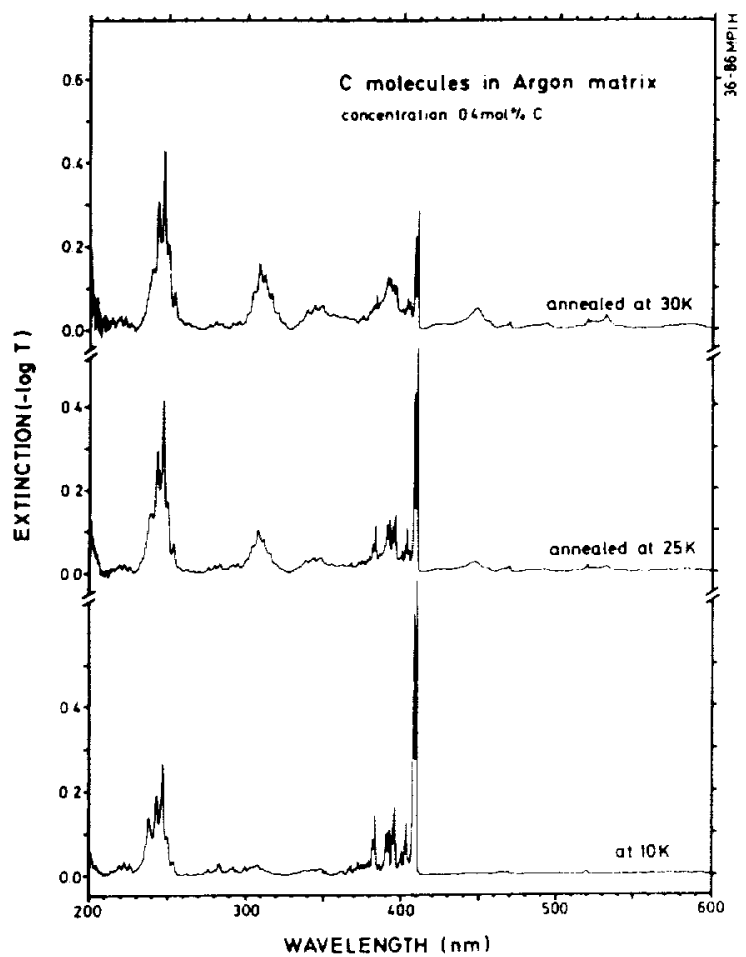

Fig. 2. The UV-Vis spectra of carbon molecules isolated in an argon matrix. 'The lower trace is of a freshly deposited sample. The middle and upper spectra were taken after the matrix had been warmed to 25 and $30 \mathrm{~K}$ respectively. The new bands produced upon warm-ap are due to the formation of larger carbon specjes (Krätschmer 1986).

Some of the species which produce the new features that lic in the visible could be DIB candidates. A comparison of the spectrum produced by this mixture of larger carbon molecules with the diffuse bands as listed by Herbig (1975) is shown in Figure 4. Disregarding the broadening, the pattern of bands in both spectra is remarkahly similar, especially between 4400 and $5600 \AA$. Interestingly, Krätschmer's $4470 \AA$ band shifts and appears quite similar to Wdowiak's $4500 \AA$ feature. Although these experiments were carried out to test the suggestion made by Douglas (1977) that linear carbon chain molecules night produce some of the DIBs. IR studies of these matrices indicate that a considerable fraction of the larger carbon molecules have nonlinear structures (Krätschuner 1986). These too must contribute to the absorptions shown in Figure 4.

In 1993, Fulara $\epsilon t$ al. reported further laboratory evidence for highly unsaturated hydrocarbons as carriers of some of the DIBs. Their results are based on an elegant experimental technique in which the UV-Vis spectra of specific mass selected species can be measured in a neon matrix. Negative $\mathrm{C}_{n} \mathrm{H}_{\mathrm{m}}$ ions (anions), where $n=2-12$ and $m<3$ arc produced in a discharge from a mixture of $10 \%$ diacetylene in argon. These were sefected out and co-deposited with neon onto the matrix substrate at 5 


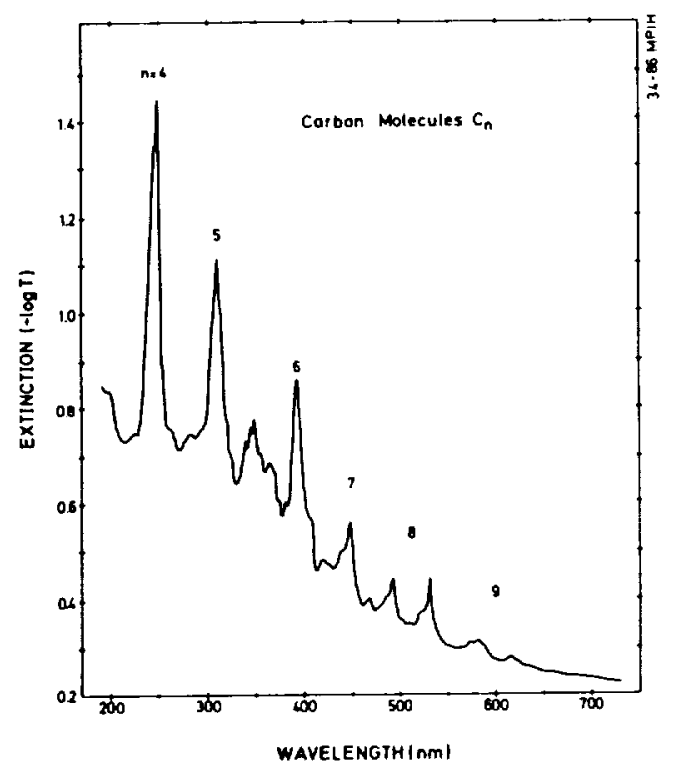

Fig. 3. The spectrum of large carbon molecules produced by annealing an argon matrix containing small carbon species to $35 \mathrm{~K}$. The bands were tentatively assigned to the $\mathrm{C}_{\mathrm{n}}$ species, $\mathrm{C}_{4}$ through $\mathrm{C}_{9}$ (Krätschmer and Nachtigall 1987).

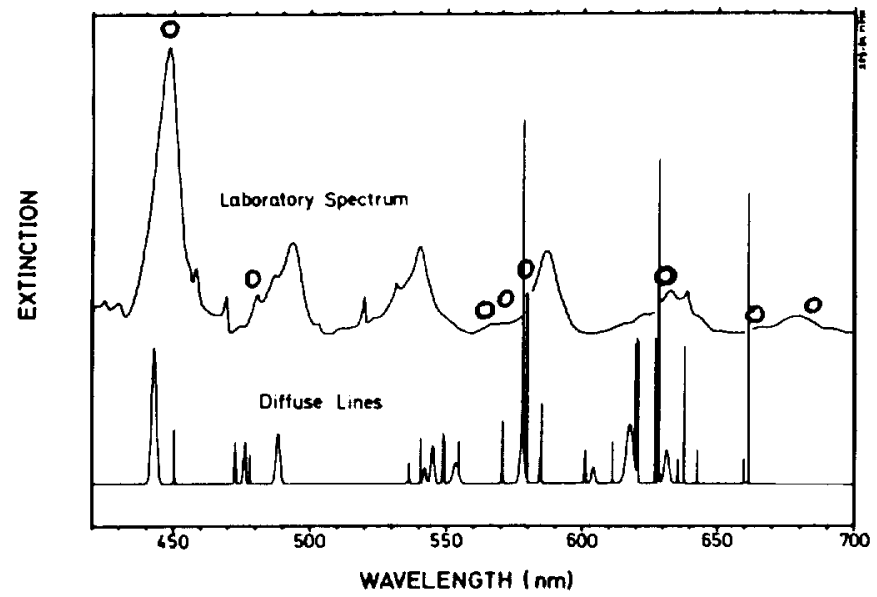

Fig. 4. Comparison of the laboratory spectrum of carbon molecules in an argon matrix with a schematic version of the DIB spectrum reported by Herbig (1975). (Fron Krätschmer 1986) 'The circles indicate the positions of the bands reported by Wdowiak (1980) in his $\mathrm{CH}_{4}$ discharge experiments. The large number of coincidences suggests that the same carriers are responsible for many of these absorptions. 


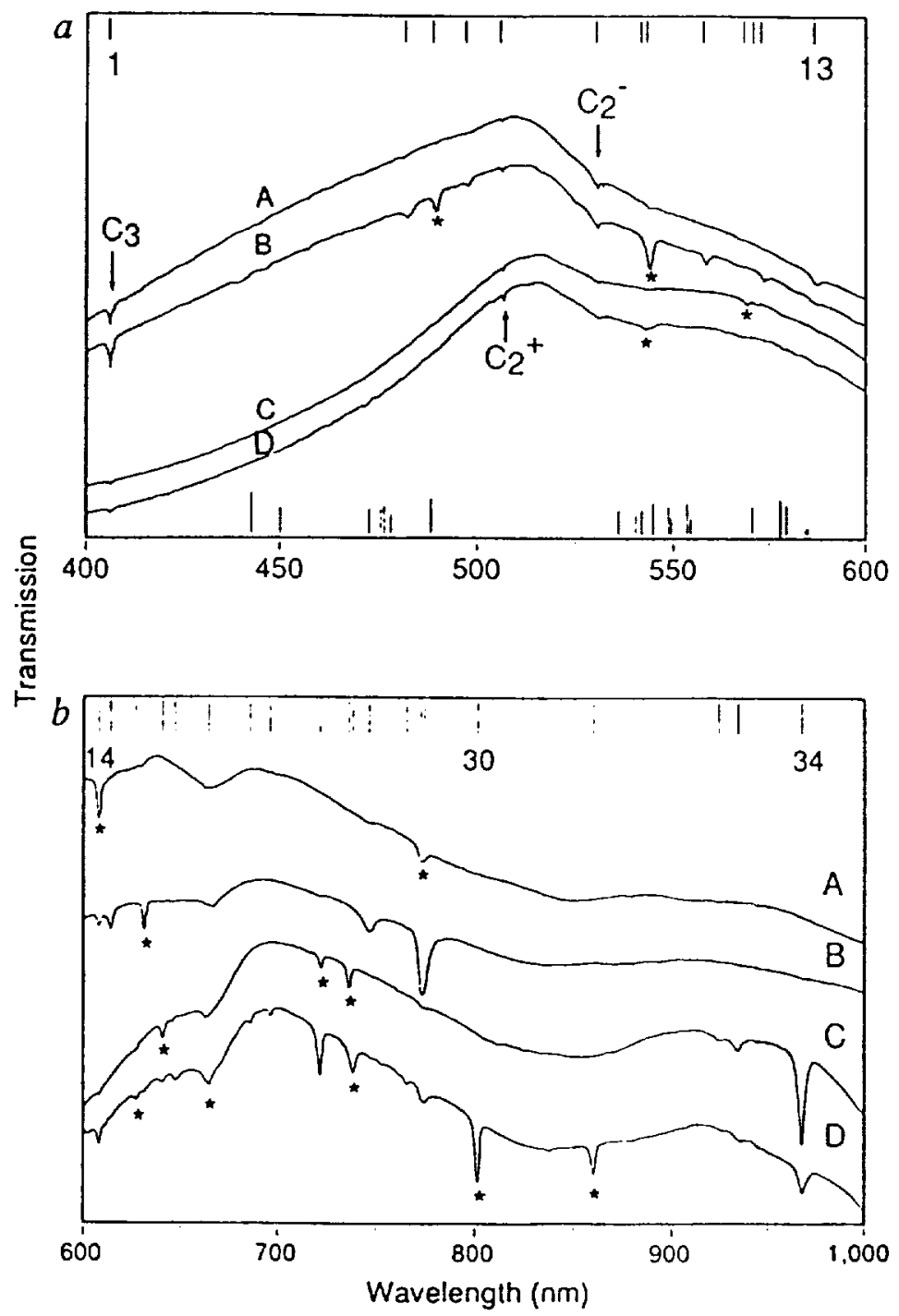

Fig. 5. The absorption spectra produced by codepositing mass-selected $\mathrm{C}_{6} \mathrm{H}_{\mathrm{m}}^{-}, \mathrm{C}_{8} \mathrm{H}_{m}^{-}, \mathrm{C}_{10} \mathrm{H}_{\mathrm{m}}^{-}$, or $\mathrm{C}_{12} \mathrm{H}_{\mathrm{m}}^{-}$and neon, with simultaneous UV irradiation (traces $\mathrm{A}, \mathrm{B}, \mathrm{C}$, and $\mathrm{D}$ ). The DIBs are shown schematically at the bottom of the upper figure, with height representing equivalent width (Herbig 1975; Herbig and Leka 1991). The observed absorption bands, numbered at the top of the figure, are listed in Table 3 . The bands labeled with asterisks lie within $36 \mathrm{~cm}^{-1}$ of known DIBs (from Fulara et al. 1993). 
K. The neon matrix was simultaneously irradiated with UV light. Figure 5 shows the absorption spectra produced by codepositing mass-selected $\mathrm{C}_{6} \mathrm{H}_{\mathrm{m}}^{-}, \mathrm{C}_{8} \mathrm{H}_{\mathrm{m}}^{-}, \mathrm{C}_{10} \mathrm{H}_{\mathrm{m}}^{-}$, and $\mathrm{C}_{12} \mathrm{H}_{\mathrm{m}}^{-}$with neon. The intensity pattern depends on deposition conditions, indicating

Table 3

Absorptions of unsaturated hydrocarbons (Figure 5) compared to the DIBs ${ }^{a}$.

\begin{tabular}{|c|c|c|c|c|}
\hline No. & & $\begin{array}{l}\lambda_{\text {matrix }} \\
(\mathrm{nm})\end{array}$ & Spectrum & $\begin{array}{c}\dot{\lambda}_{\mathrm{DIB}} \\
(\mathrm{nm})\end{array}$ \\
\hline$\frac{1}{2}$ & & $\begin{array}{r}405.8 \\
\text { (2) } 4819\end{array}$ & $\begin{array}{l}\mathrm{C}_{3} ; \mathrm{A}, \mathrm{B}, \mathrm{C}, \mathrm{O} \\
\mathrm{B}\end{array}$ & \\
\hline 3 & & (a) 489.0 & $\mathrm{~B}$ & 488.20 \\
\hline 4 & & (a) 497.5 & $B$ & \\
\hline 5 & & 506.2 & $\mathrm{C}_{2}{ }^{-} ; \mathrm{B}, \mathrm{C}, \mathrm{D}$ & \\
\hline 6 & & (e) 530.6 & $\mathrm{C}_{2}: \mathrm{A}, \mathrm{B}, \mathrm{D}$ & \\
\hline 7 & & (a) 541.9 & 0 & 542.00 \\
\hline 8 & & 543.5 & B, A, D & 544.90 \\
\hline 9 & $\#$ & 557.8 & 8 & \\
\hline 10 & $\#$ & a 568.4 & $c$ & \\
\hline 11 & & 570.8 & C & 570.51 \\
\hline 12 & & 572.8 & $B$ & \\
\hline 13 & & 586.5 & $A$ & \\
\hline 14 & & 607.7 & A, 8, C. D & 608.97 \\
\hline 15 & & 614.1 & 8 & \\
\hline 16 & $\#$ & (c) 627.1 & 0 & 626.97 \\
\hline 17 & & e 631.2 & B, 0 & 631.80 \\
\hline 18 & & a 640.7 & c. $D$ & $\left.\begin{array}{l}639.69 \\
641.26\end{array}\right)$ \\
\hline 19 & & 647.2 & $D$ & \\
\hline 20 & & (a) 664.6 & 0 & 666.07 \\
\hline 21 & {$[\#]$} & (a) 685.9 & 0 & \\
\hline 22 & & 696.0 & $D$ & \\
\hline 23 & [\#] & 721.9 & D. C & 722.40 \\
\hline 24 & & 736.4 & $c$ & 735.75 \\
\hline 25 & & 738.3 & 0 & 736.70 \\
\hline 26 & & 746.6 & $B$ & \\
\hline 27 & & 765.5 & 0 & \\
\hline 28 & & 772.8 & B. A & 772.17 \\
\hline 29 & & 774.5 & $\mathrm{D}$ & \\
\hline 30 & & 801.9 & $D$ & 802.62 \\
\hline 31 & & 860.7 & 0 & 862.08 \\
\hline 32 & & 924.6 & C. D & \\
\hline 33 & & 934.4 & C. $D$ & \\
\hline 34 & & 967.6 & c. 0 & \\
\hline
\end{tabular}

a Taken from Fulara et al. (1993). The numbers in the first column correspond to the vertical lines at the top of Figure 5 which indicate the position of a laboratory band in one of the spectra. A, B, C, and D indicate the particular spectrum shown in Figure 5 which have the band. Those absorptions labeled with [\#] are close to bands reported by Wdowiak (1980), and those with [@] are close to bands reported by Krätschmer (1986). 
that the absorptions arise from many different species. Bands marked with asterisks correlate well with DIB positions. Fulara et al. propose that the species which produce the absorptions are highly unsaturated hydrocarbon radicals, $\mathrm{C}_{\mathrm{n}} \mathrm{H}_{\mathrm{m}}$, because the anions are believed to be neutralized by the UV irradiation. On the basis of the very good agreement with DIB positions listed in Table 3 , they conclude that these species with carbon numbers between 6 and 12 may be the carriers of some of the DIBs. Smaller species are readily photofragmented, while larger structures arrange themselves into cyclic forms (see also Maier, elsewhere in this volume).

It is very important to determine oscillator strengths ( $f$-values) of the transitions in these species. Oscillator strengths are needed to calculate the column density of each proposed DIB candidate implied by the observed equivalent width. This, in turn, allows one to determine the fraction of the elemental cosmic abundance consumed by the candidate and decide whether or not it is reasonable.

\subsection{POLYCYCLIC AROMATIC HYDROCARBONS}

In 1968 Donn considered the possible contributions of individual polycyclic aromatic: hydrocarbons (PAHs) to the interstellar extinction curve. In discussing the influence of size, he pointed out that below about $200 \AA$, molecular rather than macroscopic effects become important and the calculated extinction curve starts to show considerable structure. Using the spectra of neutral, compact PAHs (presumably suspended in pellets or solution) such as coronene, ovalene and hexabenzocoronene it was argued that some of the poorly understood general features of the interstellar extinction curve - such as the high UV absorption - could be accounted for. Donn and Krishna Swamy (1969) showed that a PAH mixture was consistent with the visible extinction as well. Donn (1968) also pointed out that, "...narrow band structure may... be introduced by the radical side-chains (on PAHs). Such...narrow absorption features may be the cause of the diffuse interstellar bands in a manner having some similarity to that proposed by Johmson (1967)."

Johnson (1967), after reviewing the DIB situation at the time, concluded that, "one is probably dealing with either very complex hydrocarbon nolecules or simpler molecules in electronic states not ordinarily achieved under normal laboratory conditions..." In 1970, Johnson reported an extensive study of possible contributors to the DIBs. This work included measuring the spectra of over fifty neutral aromatic compounds suspended in various media. He concluded that "These studies do not rule out the existence of molecules of Tables IV (aromatic hydrocarbons) and V (porphyrins) entirely, however they do preclude their discovery in the optical region of the spectrum with the sensitivity presently available to astronomers." This conclusion is based on the well-known absorption property of neutral PAHs that the long wavelength absorption band limit is determined by the long dimension of the molecule (Platt, 1956; Clar, 1972). Thus, although all PAHs absorl, strongly in the UV, only larger PAHs with more than five or six rings linearly arranged will atsorb in the visible. To span the entire visible and near-infrared range, the region in which most DIBs lie, very large neutral PAHs are required. Neutral PAHs containing between 25 to 30 carbon atoms just start to absorb in the visible. Much higher numbers are required for absorptions in the red (e.g. see Salama et al. 1995 olsewhere in this volume). Consequently, since PAHs are extremely complex molecules by interstellar standards. they were not seriously considered further as DIB carriers. This conclusion that small neutral PAHs cannot contribute to the DIBs has been confirmed by the experiments of Ehrenfreund et al. (1992a) on the visible absorption spectra of the PAHs sublimed 
from coal pitch and isolated in a neon matrix. This mixture of about 150 aromatic molecules has only a few transitions in the region between 3800 and $5000 \AA$.

Since, on the other hand, PAH ions of all sizes absorb in the visible and near IR, this situation changed when it was recognized that PAHs might account for the interstellar infrared bands emitted from many different astronomical objects, and that a large fraction of any PAHs present in the diffuse interstellar medium would be ionized (Allamandola, Tielens, and Barker 1985; van der Zwet and Allamandola 1985; Léger and d'Hendecourt 1985; Crawford, Tielens, and Allamandola 1985). Spectra of many ionized PAHs suspended in boric acid glass, alkali halide, and freon matrices have been available for some time (Hoijtink 1959; Hoijtink, Velthorst, and Zandstra 1960; Shida and Iwata 1973). More recently argon matrix data on several ionized PAHs have also been published (Andrews, Kelsall, and Blankenship 1982; Andrews, Friedman, and Kelsall 1985; Szczepanski et al., 1993a,b; Vala et al. 1994). Although this work provided early support for the notion that ionized PAHs could contribute to the DIB spectrum, as described above in Section 2 - Experimental Techniques, these matrices cause spectral shifts which preclude definitive comparisons with the DIBs. Thus, since little information was available concerning the spectroscopic properties of ionized PAHs in the visible and near-infrared, new experimental studies on the electronic spectra of ionized PAHs were needed. To meet this need, a major effort was initiated at several laboratories to obtain the spectra of ionized PAHs in neon matrices.

The first comparisons of the DIBs with neon matrix spectra of PAH ions appeared only recently. These data are summarized in Table 4 (sce Salama ct al. 1995 for a detailed discussion of these data). Salama and Allamandola (1991, 1992a) reported studies of the electronic absorption spectrum of the naphthalene cation $\left(\mathrm{C}_{10} \mathrm{H}_{8}^{+}\right)$isolated in a neon matrix. The visible portion is shown in Figure 6. Neutral naphthalene does not absorb at wavelengths longer than about $3000 \AA$. The strongest band, that at $6742 \AA$, falls close to the DIB at $6741 \AA$ which was first suggested by Herbig in 1975 and confirmed by Jenniskens and Désert in 1994. The second strongest naphthalene cation band falls at $6520 \AA$. A new DIB was subsequently found at this wavelength (Table 4), although with a different strength than expected. Ehrenfreund tt al. (1992b) measured the spectra of neutral and ionized ovalene $\left(\mathrm{C}_{32} \mathrm{H}_{14}\right)$ and coronene $\left(\mathrm{C}_{24} \mathrm{H}_{12}\right)$ in neon matrices as these species were known to absorb near the strong $4430 \AA$ DIB. While these spectra showed that neither neutral ovalene nor the coronene cation could account for the $4430 \AA \mathrm{DIB}$, as shown in Table 4, the coronene cation could be responsible for the 4595 and $9466 \AA$ DIBs. Salama and Allamandola (1992b) showed that a pyrene-like cation might well be responsible for the strong $4430 \AA \mathrm{DIB}$. This was based on the observation that the pyrene cation $\left(\mathrm{C}_{16} \mathrm{H}_{10}^{+}\right)$has only one strong absorption in the entire visible and near-infrared spectral range. This band, also shown in Figurc 6, falls at $4435 \AA$ in an argon matrix and at $4390 \AA$ in neon. The difference between the DIB wavelength and the wavelength in a neon matrix casts doubt on the assignment to the pyrene cation itself. However, the proximity to the $4430 \AA$ DIB and simplicity of the pyrene cation spectrum suggested that a closely related molecule such as alkyl substituted pyrenes might prove to be strong candidates (Salania and Allamaundola 1992b). Léger and d'Hendecourt (1995, elsewhere in this volume), show that this is indeed plausible. This point is also discussed further by Salama $f t$ al. (1995, elsewhere in this volume). 

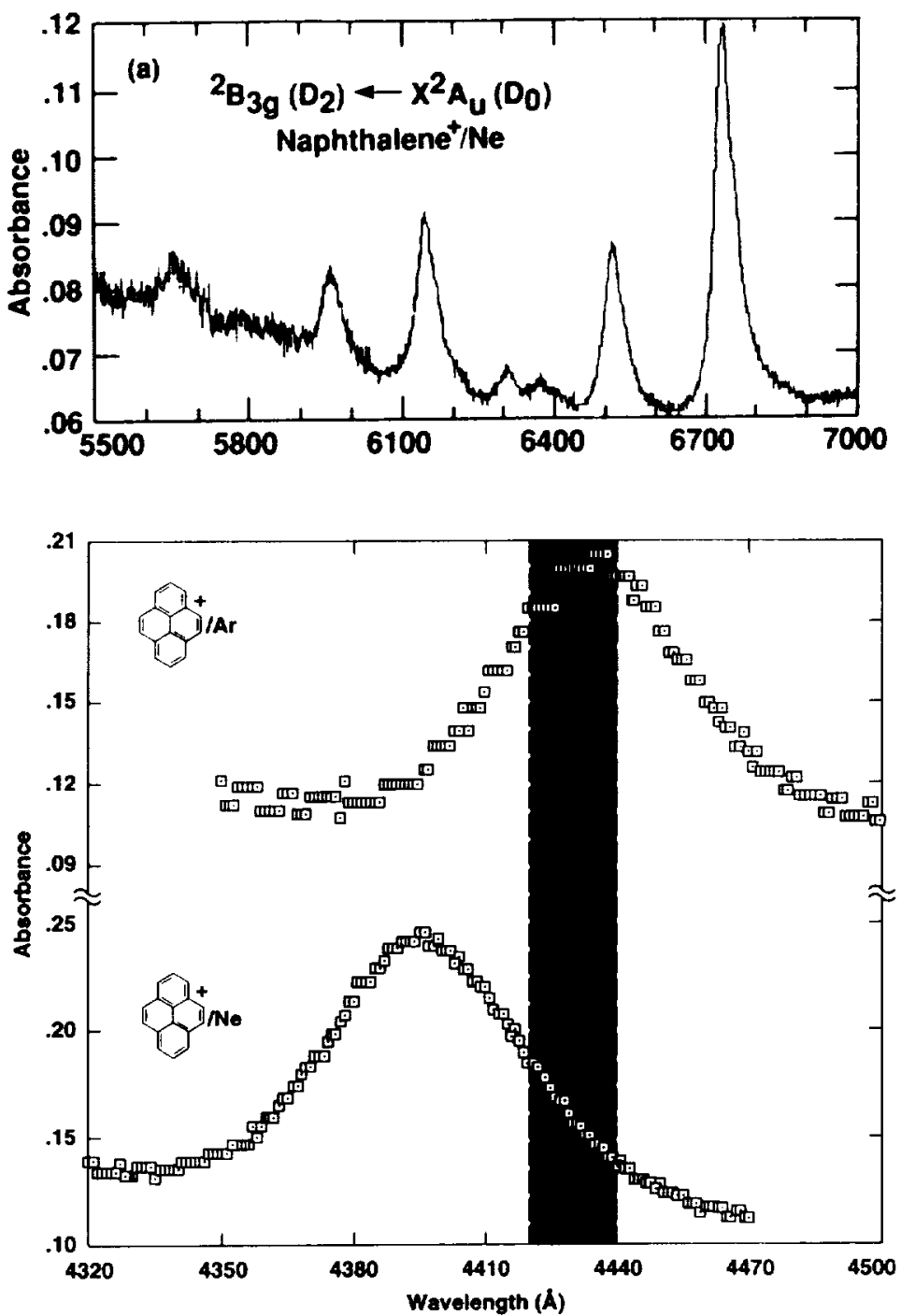

Fig. 6. Top - The visible absorption spectrum of the naphtahlene cation isolated in a neon matrix. The ion was produced by irradiating a naphthalene:neon (1:600) matrix at $12 \mathrm{~K}$ (Salama and Allamandola 1991, 1992a). Bottom - The strong UV absorption band of the pyrene cation isolated in neon and argon matrices compared to the position and width of the $4430 \AA$ DIB (shaded area, figure from Salama and Allamandola 1992b). 
Table 4

Absorption of PAH cations compared with the DIBs (Salama et al. 1995) ${ }^{a}$

\begin{tabular}{|l|l|l|c|}
\hline \hline$\overline{P A} \bar{H}^{+}$ & $\begin{array}{l}\lambda_{\text {peak }}(\mathrm{nm}) \\
(\text { Neon matrix) }\end{array}$ & DIBs $(\mathrm{nm})^{b}$ & $\Delta \nu\left(\mathrm{cm}^{-1}\right)$ \\
\hline Pyrene $\left(C_{16} H_{10}^{+}\right)$ & $439.5^{f}$ & 442.9 & 175 \\
1-Methylpyrene $\left(C H_{3}-C_{16} H_{9}^{+}\right)^{c}$ & 4443.0 in Ar) & & \\
4-Methylpyrene $\left(C H_{3}-C_{16} H_{9}^{+}\right)$ & $(457.7)$ & 442.9 & 66 \\
& 482.8 & 482.4 & 17 \\
Naphthalene $\left(C_{10} H_{8}^{+}\right)$ & 757.6 & 758.1 & 9 \\
& $674.2^{f}$ & 674.1 & 2 \\
Phenanthrene $\left(C_{14} H_{10}^{+}\right)^{d}$ & 652.0 & 652.0 & 0 \\
& $898.3^{f}$ & & 5 \\
Tetracene $\left(C_{18} H_{12}^{+}\right)$ & 856.8 & 857.2 & 2 \\
Benzo(ghi)perylene $\left(C_{22} H_{12}^{+}\right)$ & $864.7^{f}$ & 864.8 & 67 \\
& $502.2^{f}$ & $503.9(?)$ & $5 ; 4$ \\
& 758.4 & $758.1 ; 758.6$ & $11 ; 8$ \\
Coronene $\left(C_{24} H_{12}^{+}\right)^{e}$ & 755.2 & $755.8(?) ; 756.2$ & 13 \\
& 794.3 & $793.5($ prob.) & 24 \\
& $459.0^{f}$ & 459.5 & 1 \\
\hline
\end{tabular}

${ }^{a}$ Except for the $4439 \AA$ band, the percent frequency shifts between the bands in the neon matrix and the DIBs are all much less than $0.5 \%$. For the $4439 \AA$ band it is $0.75 \%$.

berbig, 1975; Sanner et al., 1978; Herbig and Leka, 1991; Jenniskens and Désert, 1994; Ehrenfreund and Foing, 1994.

'See also Léger and d'Hendecourt, 1995.

d Phenanthrene data from Salama et al. 1994.

e See also Ehrenfreund et al., 1992.

f Strongest absorption band.

The oscillator strengths determined for these species permit one to calculate the abundance of the PAH candidate required to account for the observed DIB equivalent width. For each of the PAHs listed in Table 4, only a few tenths of a percent of the cosmic carbon is implied, showing that PAHs readily satisfy the cosmic abundance constraint.

An important question related to the issue of PAHs as DIB carriers has to do with the UV spectral properties of these species. Since neutral PAHs are known to 
possess strong absorptions in the UV, the lack of substructure in the UV portion of the interstellar extinction curve has been considered as a criticism of the PAH hypothesis (see Donn 1995, elsewhere in this volume). There are two factors that would tend to weaken this substructure. First, the absorptions produced by a mixture of PAHs would overlap, producing only a weakly structured pseudocontinuum in a manner similar to that described by Joblin et al. (1992). Second, the UV band strengths of some PAH ions may be weaker than for their neutral counterparts. The UV absorption strength of the naphthalene cation appears to be weaker than for neutral naphthalene (Salama and Allamandola 1991, 1992a). This tendency has also been found for the cations of several PAHs suspended in borosilicate glasses (Lee and Wdowiak 1993). If this is a general property of ionized PAHs, the implication is that ionized interstellar PAHs might not add significant structure to the interstellar extinction curve at wavelengths shortward of about $4000 \AA$ along lines-of-sight with strong DIBs. The ionization state of a mixture of PAHs is expected to vary in the diffuse interstellar medium (Bakes and Tielens, elsewhere in this volume). If ionized PAHs indeed contribute to the DIBs, it would be interesting to search for UV structure in those regions where DIBs are known to weaken. Since the PAH spectrum is such a sensitive function of ionization state, as the population of ionized PAHs decreases, UV absorption bands due to the neutral species may become apparent.

\subsection{FULLERENES AND RELATED MATERIALS}

A spell was cast over much of the astronomical community when Kroto et al. (1985) suggested that $C_{60}$ had the structure of a soccerball and that "it may be widely distributed throughout the Universe". Kroto et al. also speculated that $\mathrm{C}_{60}$ or a derivative may be the carrier of the DIBs. As with PAHs, there is a need for the spectroscopic properties of these species taken under conditions appropriate for the interstellar medium. During the past few ycars some of this data has become available.

The 2000 to $7000 \AA$ spectrum of neutral $C_{60}$ in solution or as a solid film has been reported by Krätschmer et al. (1990) and Leach et al. (1992). $C_{60}$ absorbs most strongly at about 2150,2600 , and $3350 \AA$, possessing very weak bands in the visible. The spectrum presented in Figure 7 shows that neutral $C_{60}$ is not a DIB candidate. However, as with PAHs, the ions of this species absorb at longer wavelengths. Recently, the spectra of matrix isolated $\mathrm{C}_{60}^{+}$and $\mathrm{C}_{60}^{-}$have become available (argon: Gasyna, Andrews, and Schatz 1992; neon: Joblin 1992; neon and argon: Fulara, Jakobi, and Maier 1993). The spectra of neon matrix isolated $\mathrm{C}_{60}^{+}$and $\mathrm{C}_{60}^{-}$ are shown in Figure 8 . The strongest absorptions of $\mathrm{C}_{60}^{+}$fall at 10368 and 10435 $\mathrm{cm}^{-1}\left(9645\right.$ and $9583 \AA$ ), while the strongest band of $\mathrm{C}_{60}^{-}$is at $9443 \mathrm{~cm}^{-1}(10590 \AA)$. While these fall beyond the spectral region normally associated with the DIBs, recent observations reported by Foing and Ehrenfreund (1994), have revealed two possible new DIBs at 9632 and $9577 \AA$. These lie within 14 and $7 \mathrm{~cm}^{-1}$ of the two strong neon matrix isolated $\mathrm{C}_{60}^{+}$bands. The relative band strengths are also similar to those found for matrix isolated $\mathrm{C}_{60}^{+}$. Thus, these new DIBs may well arise from $\mathrm{C}_{60}^{+}$(Foing and Ehrenfreund 1994; and Foing and Ehrenfreund 1995, elsewhere in this volume). Questions remain however as the doublet structure shown in Figure 8 may arise from $\mathrm{C}_{60}^{+}$being trapped in two different forms (not sites), one metastable, by the matrix. Gas phase $\mathrm{C}_{60}^{+}$may not show this doublet structure. Since any DIB identification is strengthened by multiple band agreements, this would weaken the $\mathrm{C}_{60}^{+}$assignment, 


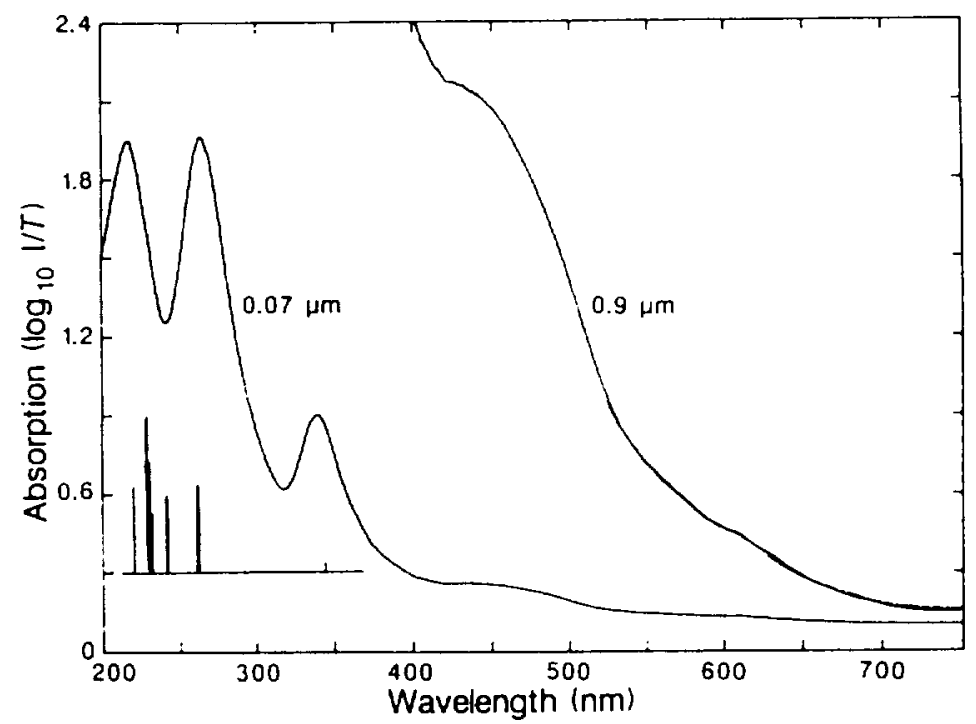

Fig. 7. The Visible-Ultraviolet absorption spectra of two thicknesses of solid, neutral $\mathrm{C}_{60}$. The stick spectrum at the bottom shows the positions and relative oscillator strengths theoretically calculated for isolated $\mathrm{C}_{60}$. Figure from Krätschmer et al. (1990).

but not eliminate it as one of the doublet components would remain. Maier (1994) has also discussed this issue.

As with the carbon chains, it is very important to determine the oscillator strengths for these transitions in order to deduce the fraction of the elemental cosmic abundance required by the candidate.

\subsection{PORPHYRINS AND RELATED MATERIALS}

In 1970 Johnson reported an extensive spectroscopic study of potential DIB candidates including dyes, sulfur containing species, aromatic compounds with and without hetero atoms, and porphyrins. This exhaustive work encompassed possible grain materials as well as individual molecules suspended in matrices of various sorts. Since the spectra on porphyrins looked promising, while the other spectra "did not even remotely suggest similarity to the diffuse interstellar spectra" a large number of porphyrin compounds were studied further. Since many porphyrins possess an unusually strong band (the Soret band) in the vicinity of $4430 \AA$, the position of the strongest DIB, Johnson considered many closely related compounds. The compound, bispyridylmagnesiumtetrabenzoporphine, (MgTBP-dipyridine), with the structure shown in Figure 9, seemed particularly promising.

As shown in Figure 9, its two strongest absorptions fall close to the two strongest DIBs, namely those at 4428 and $6281 \AA$. The bands are broad as are the two strong DIBs. Sixteen spectral coincidences with known DIBs were reported at that time, with six others added later (Johnson, Bailey, and Wegner 1973). Johnson (1991; 


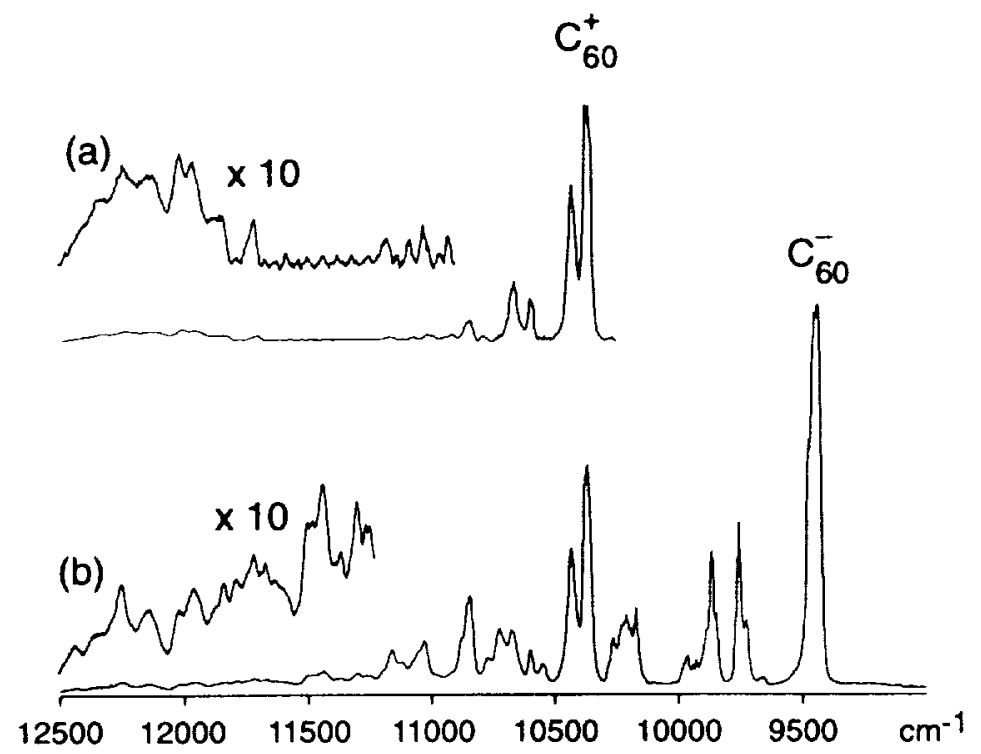

Fig. 8. The electronic absorption spectra of $\mathrm{C}_{60}^{+}$and $\mathrm{C}_{60}^{-}$isolated in neon matrices. Figure from Fulara, Jakobi, and Maier (1993).

1995, elsewhere in this volume) reports that most of the DIBs can be reproduced by the spectra obtained when this compound is suspended in several different paraffin matrices.

The novel suggestion that porphyrins or closely related materials might account for the DIBs was recently furthered by Miles and Sarre (1992), when they pointed out that the strong origin band of cold, gas phase chlorin $\left(\mathrm{C}_{20} \mathrm{H}_{16} \mathrm{~N}_{4}\right)$, a reduced porphyrin, falls within $1 \AA$ of the prominent DIB at $6284 \AA$. Miles and Sarre (1993) discussed additional spectroscopic and analytical studies of this species which supports the notion of chlorin as a DIB carrier. Figure 10 which compares a schematized version of the DIB spectrum with measured and calculated chlorin spectra shows the suggestive nature of this assignment. In their thorough discussion of this suggestion, Mifes and Sarre (1993) also point out that the very strong and broad Soret band which falls near $3900 \AA$ must be searched for to test this assignment and that currently available data are of insufficient quality to settle the issue. See Hibbins et al. 1994, for a discussion of new, narrow DIBs detected in this region.

\section{ASSESSMENTS}

Here, a brief assessment of the status of each suggested material is presented, with emphasis placed on what is needed to really put each candidate to the test. Limitations are also discussed.

UNSPECIFIED ORGANICS

The specific materials responsible must be identified, or at the very least placed 


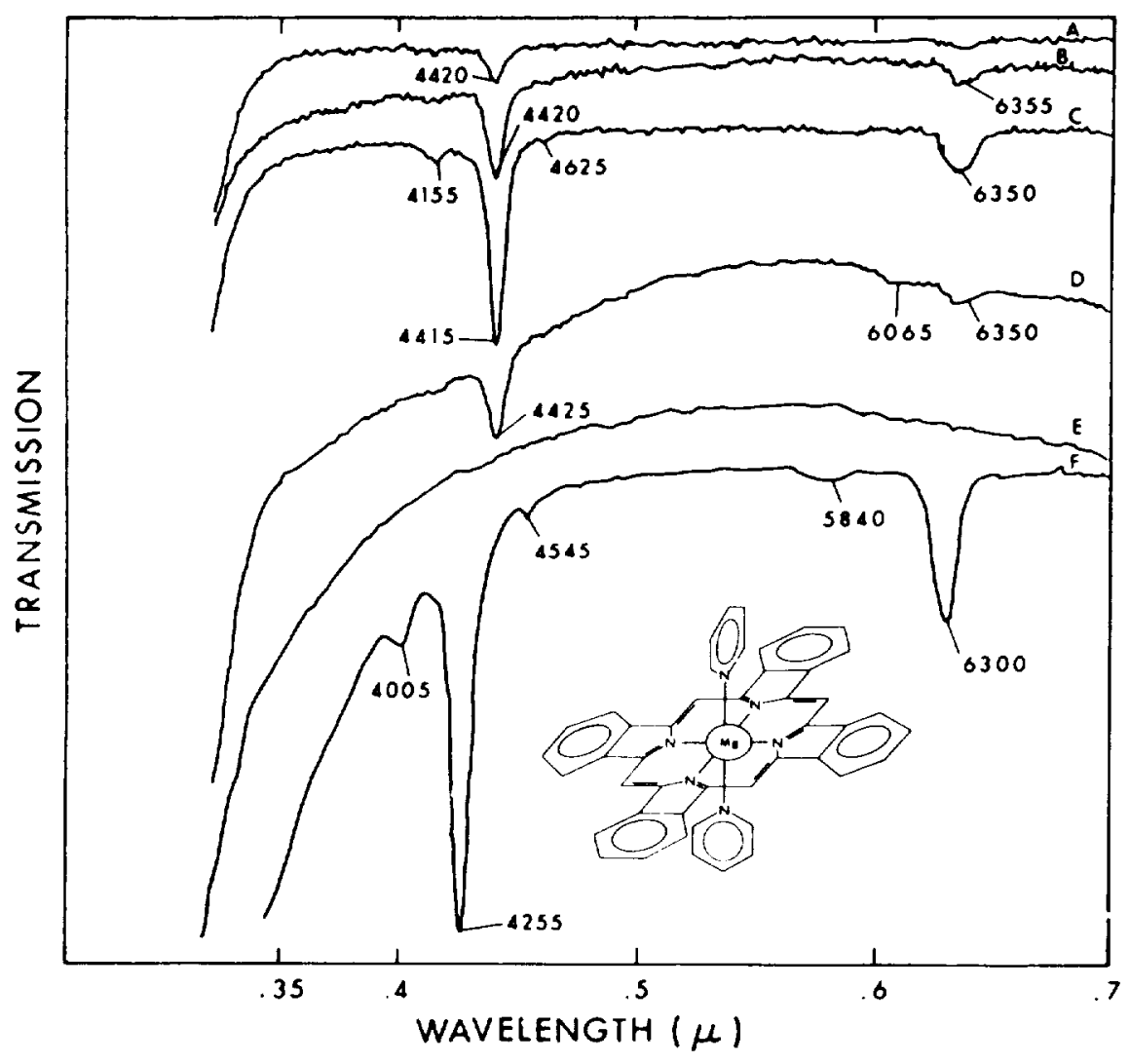

Fig. 9. The 3500 to $7000 \AA$ spectrum of Bispyridylmagnesiumtetrabenzoporphine dissolved in pyridine as a function of pathlength $(A, B, C)$ and concentration ( $A$ and $D)$. E and $F$ are traces of pyridine alone, and the compound dissolved in TEA. Figure from Johnson (1970). 


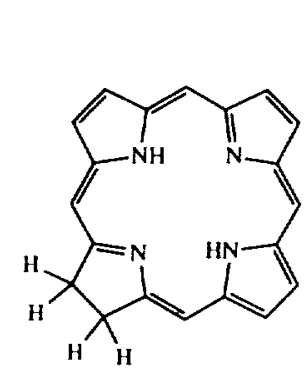

(c)
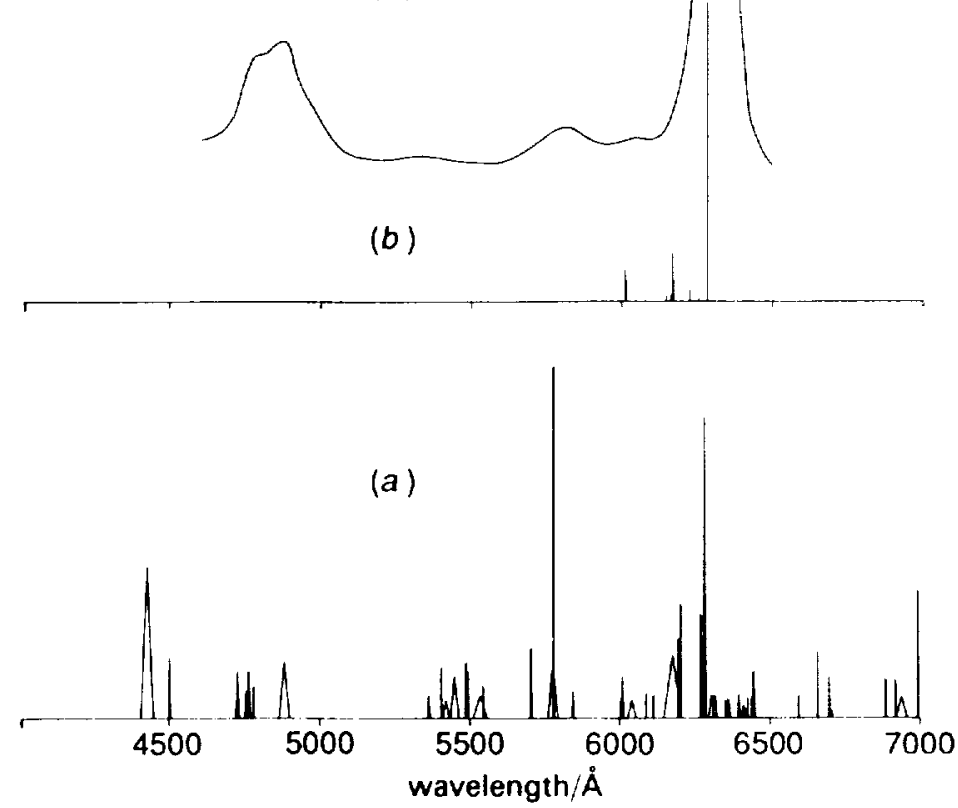

Fig. 10. (a) - A schematic and simplified version of the DIBs compared with (b) - the fluorescence spectrum of gas-phase, jet-cooled chlorin, and (c) the spectrum of chlorin in benzene solution at room temperature, shifted by $-91 \AA$. From Miles and Sarre (1992). 
in general chemical categories. To achieve this will require new experiments carried out at the low end of the ultra-high vacuum range to eliminate any questions of contamination, allowing a confident identification of the elements involved. Once this is achieved, one can then compare the properties of these materials with the properties of the other candidates. One key question at this stage is whether or not these materials are the same as, or closely related to, those generated in the carbon chain studies. Once classification has been made, studies in neon matrices will be required to see if the new bands still fall within $1 \%$ of the DIB wavelengths. If so,

- $f$-values must be determined. If all of these criteria are met, gas phase experiments, on a least a few representative samples, will be called for.

\section{CARBON CHAINS}

In view of the encouraging neon matrix results, these species look quite promising as candidates for some of the DIBs. It is important to increase the spectral library of these species and determine oscillator strengths ( $f$-values). The most promising candidates warrant the work needed to determine the specific molecular formulae for several reasons. First, this will provide guidance for the gas phase experimental work called for to verify band positions; second, specific identifications will allow theoretical calculations on gas phase band strengths, profiles, and relative intensities; and third, the species suggested will give much needed insight into the chemical and physical processes of the interstellar medium. It presently seems that while carbon chains might account for some of the DIBs, they probably cannot account for them all since each species is likely to produce a few bands at most . This constraint appears fairly tight because small species ( $\leqslant 6 \mathrm{C}$ atoms) are not photostable under the interstellar radiation field, while larger species ( $\gtrsim 10-12 \mathrm{C}$ atoms) cyclize into aromatic moities. Even allowing for mixtures of slightly larger isomers, it is difficult to imagine such a handful of species producing all of the DIBs.

\section{POLYCYCLIC AROMATIC HYDROCARBONS}

In view of the encouraging neon matrix results, these too, look very promising as DIB candidates. It is important to increase the spectral library of neon matrix isolated PAHs, not only as positive and negative ions, but also in their radical forms. The $f$-values are extremely important to determine as these are used to derive column densities and there is great uncertainty in the current experimental literature on this topic. Gas phase spectroscopic work is called for on the most promising species to verify band positions and calculated profiles. While it appears that PAHs might well account for some of the DIBs, they suffer from a concern which is the opposite to that for carbon chains. There are so many PAH isomers conceivable that one must envision a mechanism which favors several tens of PAHs, while weeding many out. Unless this is the case, the cosmic carbon available must be distributed among all PAHs, lowering the column density of any specific PAH to the point where it cannot absorb strongly enough to produce a typical DIB equivalent width. Since different PAHs have widely varying stabilities, weeding out may occur during formation in circumstellar shells or in the interstellar medium itself.

\section{$\mathrm{C}_{60}$ AND FRIENDS}

While there is no spectroscopic evidence in support of $\mathrm{C}_{60}$ as an abundant interstellar species (due to the lack of structure in the $2000-3000 \AA$ portion of the extinction curve), there is intriguing agreement between the two strongest absorption bands in the spectrum of neon matrix isolated $\mathrm{C}_{60}^{+}$and two newly detected DIBs. To test this further requires that the gas phase spectrum of $\mathrm{C}_{60}^{+}$be measured and that $\mathrm{f}$-values be determined. Observationally, correlation studies demonstrating that the two new DIBs are locked together are also required to test the identification. To determine if 
$\mathrm{C}_{60}^{-}$is present requires the search for a new DIB in the vicinity of $10590 \AA$, the wavelength of its strongest absorption. In any event, neither $\mathrm{C}_{60}, \mathrm{C}_{60}^{+}$, nor $\mathrm{C}_{60}^{-}$contribute to most of the known DIBs. See Kroto (1988) for a discussion of various other conceivable forms of fullerenes which may be considered for the DIBs, including metal entrapped species, and Webster (1995, elsewhere in this volume) for a discussion of the many hypothetical variations on the $\mathrm{C}_{60}$ theme such as hydrogen additions at various positions on the molecule which can isolate conjugated bond systems and create radical sites, providing systems analogous to polyenes such as polyethylene which can absorb in the visible.

\section{PORPHYRINS AND RELATED MATERIALS}

This intriguing suggestion, which has been proposed to account for the DIBs and UV-Visible extinction, involves the suspension of MgTBP-dipyridine in 5 types of paraffin matrices. For this suggestion to be widely accepted will require that all of the spectra be published over the entire spectral range considered. Band positions, intrinsic strengths ( $f$-values), and relative strengths need to be tabulated as well. Although the picture involves MgTBP-dipyridine suspended in paraffin, gas phase data, especially for the very intense Soret band, is also needed. This is in addition to spectra of this compound in or on other interstellar materials known since it is difficult to imagine that this molecule would only be present in paraffin in the interstellar medium. These data are needed to fully test this DIB candidate as it would provide a tell-tale tracer for this compound prior to paraffin incorporation. In addition, the infrared spectra of the paraffin/MgTBP-dipyridine suspensions should also be published in order to provide spectral signatures with which the presumably more abundant paraffin (and possibly MgTBP-dipyridine) can be searched for. Although there is currently little data on the infrared spectral properties of the diffuse interstellar medium the upcoming $I S O$ mission should address this issue squarely. For chlorin, a complete UV-Visible spectrum in a neon matrix or in the gas-phase (including $f$-values) is needed to pin down the other band positions.

Finally, although it almost goes without saying, the species considered must meet a number of criteria in addition to spectral matching since there are so many DIBs that apparent wavelength agreement, while crucial, should only be viewed as the first hurdle. For example, the species must satisfy cosmic abundance constraints and they must be reasonable. The later point is important because of the seductive power of the DIB problem to spectroscopists. This has often led to the vigorous pursuit and development of unreasonable ideas. The following serves to illustrate the pitfalls of relying solely on wavelength comparisons. Some time ago the author (and one of the editors!) expended a very serious experimental and theoretical effort on the study of simple radicals such as $\mathrm{HCO}$ and $\mathrm{NH}_{2}$ frozen in various matrices. The motivation was the remarkably close agreement between a number of the strongest DIBs reported by Herbig (1975) and the strongest absorption bands of these frozen radicals. The strongest absorption band wavelengths of each species are listed, followed by possible DIB counterparts in parentheses. The first DIB wavelength is from Herbig (1975), the second from Jenniskens and Désert (1994). HCO in CO: $5102 \AA$ $(-, 5109 \AA), 5330 \AA(5362 \AA, 5362 \AA), 5548 \AA(5545 \AA, 5545 \AA), 5789 \AA(5778 \AA, 5789 \AA)$, $6052 \AA(6042 \AA, 6045 \AA) 6352 \AA(6352 \AA, 6354 \AA), 6695 \AA(6660 \AA, 6699 \AA)$ [HCO data from Ewing, Thompson, and Pimentel 1960]; $\mathrm{NH}_{2}$ in argon: $4711 \AA$ (4726 $\AA, 4727 \AA$ ), $5150 \AA(-,-), 5684(5705 \AA, 5705 \AA), 6275(6270 \AA, 6278 \AA)\left[\mathrm{NH}_{2}\right.$ data from Robinson and McCarthy 1959)]. This work was pursued notwithstanding the fact that this "wavelength agreement" necessitated the use of different matrices - CO and Ar; both of which are absurd for the diffuse interstellar medium (matrices were rationalized 
for two reasons, first to shift the wavelengths into agreement since many of the gas phase values were off by more than a hundred angstroms, and second to protect these fragile species from photodissociation by the interstellar radiation field). Fortunately, we came to our senses in time.

\section{CONCLUSION}

- Spectroscopic studies of potential diffuse interstellar band carriers are reviewed. The discussion is limited to the spectroscopic properties of those species thought to be promising by various workers in this field. These include - unspecified organics, carbon chains, polycyclic aromatic hydrocarbons (PAHs), fullerenes and derivatives, as well as porphyrins and related material. A critical assessment of the status of each material as a potential DIB candidate is given, along with suggestions for further experimental studies needed. In addition to the spectra discussed here, many other properties of the DIB candidates are required to fully test the feasibility of each.

The criteria which must be met for a particular material to be accepted as a DIB carrier are that its visible and near-infrared absorption features match the known DIBs in wavelength, bandwidth, and relative intensities, while not possessing additional features which are absent in the interstellar spectra. There are no species which presently satisfy these criteria. At this point given the type and quality of published data available, carbon chains and PAHs are the most promising candidates to account for a number of the DIBs.

ACKNOWLEDGEMENTS. I wish to thank both Xander Tielens and Ted Snow for their extreme patience and understanding while I prepared this manuscript. I also wish to thank Farid Salama for his careful reading of the manuscript. This work was supported by NASA's Infrared, Submillimeter, and Radio Laboratory Astrophysics Program (Grant 188-44-57-01), NASA's Long Term Space Astrophysics Research Program (Grant 399-20-00-05), and NASA's Ultraviolet, Visible, and Gravitational Astrophysics Research and Analysis Program (Grant 188-41-57-41).

\section{REFERENCES}

Allamandola, L.J. Tielens, A.G.G.M., and Barker, J.R. 1985, Ap. J. (Letters), 290, L25.

Andrews, L., Friedman, R.S., and Keisall, B.J. 1985 J. Phys. Chem., $\mathbf{8 9}, 4016$

Andrews, L., Kelsall, B.J., and Blankenship, T.A. 1982, J. Phys. Chem., 86, 2916

Bakes E. and Tielens, A.G.G.M. 1995, in The Diffuse Interstellar Bands, eds. A.G.G.M. Tielens and T.P. Snow (Kluwer, Dordrecht), this volume.

Boissel, P. 1995, in The Diffuse Interstellar Bands, eds. A.G.G.M. Tielens and T.P. Snow (Kluwer, Dordrecht), this volume.

Bondybey, V. E. and Miller, T.A. 1983, in Molecular lons: Spectroscopy, Structure and Chemistry, eds. T.A. Miller and V.E. Bondybey, (North Holland Publishers), 125.

Clar, E. 1972, The Aromatic Sextet, (London, John Wiley and Sons).

Crawford, M.K., Tielens, A.G.G.M., and Allamandola, L.J. 1985, Ap. J. (Letters), 293, L48.

Donn, B. 1968, A p. J., 152, L129

Donn, B. and Krishna Swamy, K.S. 1969, Physica, 41, 144.

Donn, B. 1995, in The Diffuse Interstellar Bands, eds. A.G.G.M. Tielens and T.P. Snow (Kluwer, Dordrecht) this volume

Douglas, A.E. 1977, Nature, 269, 130

Ewing, G.E., Thompson, W.E., and Pimentel, G.C. 1960, J. Chem. Phys., 32, 927. 
Ehrenfreund, P., d'Hendecourt, L.B., Joblin, C., and Léger, A. 1992a $A \mathcal{E} A, \mathbf{2 6 6} 429$

Ehrenfreund, P., d'Hendecourt, L.B., Verstraete, L., Léger, A., Schmidt, W., and Defourneau, D $1992 \mathrm{~b}, A \& \&$ 259, 257

Ehrenfreund, P. and Foing, B.H. 1994, (private communication).

Foing, B. and Ehrenfreund, P. 1995, in The Diffuse Interstellar Bands, eds. A.G.G.M. Tielens and T.P. Snow (Kluwer, Dordrecht), this volume.

Foing $\mathrm{B}$ and Ehrenfreund P 1994 , Nature, $\mathbf{3 6 9}, 296$

Foing, B. and Jakobi, M., and Maier, J.P. 1993, Chem. Phys. Lett, 211 227.

Fulara, J., Lessen, P., Freivogel, P., and Maier, J.P. 1993, Nature, 366, 439

Gasyna, Z. Andrews, L., and Schatz, P.N. 1992, J. Phys. Chem., 96, 1525.

Herbig, G.H. 1975, Ap. J, 196, 129

Herbig, G.H, and Leka, K.D. 1991 A A. J., 382, 193.

Hibbins, R.E., Miles, J.R., Sarre, P.J., and Herbig G.H. 1994, in The Diffuse Interstellar Bands: Contributed Papers, eds. A.G.G.M. Tielens, (NASA CP 10144), 31

Hoijtink, G.J. 1959 Molecular. Phys., 2, 85.

Hoijtink, G.J., Velthorst, N.H., and Zandstra, P.J. 1960, Molecular. Phys., 3, 533

Jenniskens, P. and Désert, F.X. $1994, A$ E A Supp. Ser., 106, 39.

Joblin, C. 1992, Ph.D. Dissertation, University of Paris 7192

Joblin, C., Léger, A., Martin, P., and Defourneau, D. 1992, in Astrochemistry of Cosmic Phenomena, ed. P.D. Singh (Kluwer:Dordrecht), 21

Johnson, F. 1967, in Interstellar Grains, eds. J.M. Greenberg and T.P. Roark (NASA SP-140), 229

Johnson, F.M. 1970, Spectroscopic Studies of Interstellar Grains, NASA CR - 1667

Johnson, F.M., Bailey, D.T., and Wegner, P.A. 1973, in Interstellar Dust and Related Topics, eds. J.M. Greenberg and H.C. van de Hulst, (Reidel, Dordrecht), 317.

Johnson, F.M. 1991, Bull. Am. Astron. Soc., 23, 933

Johnson, F.M. 1995, in The Diffuse Interstellar Bands, eds. A.C.(A.M. Tielens and T.P. Snow (Kluwer, Dordrecht), this volume.

Krätschmer, W. 1986, Ap. 60 Sp.Sci. 128,93

Krätschmer, W. Lamb, L.D. . Fostiropoulos, K., and Huffman, D.R. 1990, Nature, 347, 354

Krätschmer, W. and Nachtigall, K. 1987 , in Polycyclic Aromatic Hydrocarbons and Astrophysics, eds. Léger, A d'Hendecourt, L.B., and Boccara, H., 75 .

Krelowski, J. and Walker, G.A.H. 1987, Ap. J., 312, 860 .

Kroto, H.W. 1988 , Science, 242,1139

Kroto, H.W. Heath J.R. O'Brien S.C Curl R.F and Smalley, R.F 1985 Nature, 318162

Leach, S. 1995, in The Diffuse Interstellar Bands, eds. A.G.G.M. Tielens and T.P. Snow (Kluwer, Dordrecht), this volume.

Leach, S., Vervloet, M., Despres, A., Breheret, E., Hare, J.P., Dennis, T.J., Kroto, H.W., Taylor, R., and Walton, D.R.M. 1992, Chem. Phys. 160451

Lee, W. and Wdowiak, T.J. $1993, A$. J. (Letters $\mathbf{4 1 0}, \mathrm{L} 127$

Léger, $\mathrm{A}$. and d'Hendecourt, L. B. $1985, A \& \mathcal{S}^{\prime} A, 146,81$ '.

Léger, A. and d'Hendecourt, L.B. 1995, in The Diffuse Interstellar Bands, eds. A.G.G.M. Tielens and T.P. Snow (Kluwer Dordrecht), this volume.

Maier, J.P. 1994, Nature, 370, 423 .

Miles, J.R., and Sarre, P.J. 1992, J. Chem. Soc. Faraday Trans., 88, 1075.

Miles, J.R. and Sarre, P.j. 1993 J. Chem. Soc. Faraday Trans., $\mathbf{8 9} 2269$.

Platt, J.R. 1956, Ap. J., 123, 486 .

Robinson, G.W. and McCarthy, M. 1959, J. Chem. Phys. 30 999

Salama, F. and Allamandola, L.J. 1991, J. Chem. Phys. 94,6964

Salama, F. and Allamandola, L.J. 1992a, $A$ p. J., 395, 301

Salama, F. and Allamandola L.J. 1992b, Nature 358, 42

Salama, F., Joblin, C., and Államandola, L.J. 1994, J. Chem. Phys. 101, 10252.

Salama, F. Joblin, C., and Allamandola, L.J. 1995, in The Diffuse Interstellar Bands, eds. A.G.G.M.Tielens and T.P. Snow (Kluwer, Dordrecht), this yolume.

Sanner, F., Snell, R, and Vanden Bout P. 1978, Ap.J., 226, 460

Shida, T. and Iwata, S. $1973, J$. Am. Chem. Soc. 95, 3473 .

Szczepanski, J., Chapo, C. and Vala M. 1993a, Chem. Phys. Lett., 205, 434

Szczepanski, J., Vala M., Talbi, D. Parisel, O., and Ellinger, Y. 1993b, J. Chem. Phys 984494

Vala, M., Szczepanski, J., Pauzat, F., Parisel, Oे., Talbi, D., and Ellinger, Y. 1994, J. Phys. Chem.,

van der Zress. G.P. and Allamandola, L.J. 1985, $A \& \mathcal{E} A, 146,76$.

Webster, A. 1995, in The Diffuse Interstellar Bands, eds. A.G.G.M. Tlelens and T.P. Snow (Kluwer, Dordrecht), this volume.

Wdowiak, T.J. 1980, Ap. J. (Letters), 241 L55.

Wdowiak, T.J., Beegle, L.W., Lee, W., and Robinson, M.S. 1994, in The Diffuse Interstellar Bands: Contributed Papers, eds. A.G.G.M. Tielens and T.P. Snow, (NASA CP 10144), 65

Wdowiak, T.J., Lee,W., and Beegle, L.W. 1994 in Molecules and Grains in Space, ed. I. Nenner, AIP Conf. Proc. 312, (Am. Inst. Phys. Press, New York), 687 